\title{
Commentary
}

\section{A New "Transgrade" Technique for Dorsal Root Ganglion Stimulation Shows Promise in Safety and Efficacy}

Timothy D. Kwiecien, MD, and Jianguo Cheng, MD, PhD

From: Departments of

Pain Management and Neurosciences, Cleveland Clinic

Cleveland, Ohio, 44195 USA

Address Correspondence: Jianguo Cheng, MD, PhD Professor and Director Cleveland Clinic Multidisciplinary

Pain Medicine Fellowship Program

Departments of Pain Management and Neurosciences Cleveland Clinic 9500 Euclid Avenue Cleveland, Ohio, 44195 E-mail: chengj@ccf.org

Disclaimer: There was no external funding in the preparation of this manuscript. Conflict of interest: Each author certifies that he or she, or a member of his or her immediate

family, has no commercial association (i.e., consultancies, stock ownership, equity interest, patent/licensing arrangements, etc.) that might pose a conflict of interest in connection with the submitted manuscript.

Manuscript received: 09-01-2019 Accepted for publication: 10-01-2019

Free full manuscript: www.painphysicianjournal.com

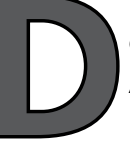
orsal root ganglion (DRG) stimulation was approved by the US Food and Drug Administration in early 2016 for the treatment of moderate to severe chronic intractable pain of the lower limbs in adult patients with complex regional pain syndrome (CRPS I and II). As more clinicians start to use this treatment modality, it becomes clear that DRG stimulation is technically more challenging compared to traditional spinal cord stimulation (SCS). The use of a guidewire and sheath for placing the electrodes in close proximity to the DRGs often causes irritation of the DRG and/or its corresponding nerve during and/or after the procedure. Adnan Al-Kaisy et al. report an alternative technique for DRG stimulation in this issue of Pain Physician (1). Also reported in the paper are the results from 39 retrospectively reviewed cases that appear to be promising in safety and efficacy. A key feature of this new technique is the use of a Racz-Coude needle for epidural access at a "transgrade" angle without requiring any specialized hardware or accessories. The authors argue that the new technique may reduce the risk of neurological complications as it does not require a guidewire and sheath. Furthermore, the path of the needle is more parallel to the foramina allowing the lead to anatomically follow the nerve. Supporting this argument, they did not report encountering any difficulty inserting the leads into the foramina despite using a larger lead and they did not report any incidences of dural puncture in this case series.

There have been several published approaches and techniques for DRG implantation. The intraspinal approach directs the stimulator lead from the epidural space to the nerve root. The transforaminal technique is similar to executing a transforaminal epidural steroid injection where the stimulator leads are placed from an outside-toinside approach as it relates to the neural foramina (2). A retrograde approach has been utilized specifically for nerve root and sacral nerve stimulation (3-5). In contrast, the transgrade approach places a lead in the neural foramina from the opposite side of the interlaminar space. The epidural needle's curve was directed in a posterior direction which aided the placement of the electrode into the neural foramina. An extended plastic introducer was employed which allowed the proceduralist to change needle bevel direction while avoiding dural puncture.

The transgrade technique for permanent implantation of DRG leads offers a novel technique for pain medicine specialists. Authors from this study cautioned that there is a learning curve involved with this particular technique and providers should obtain sufficient clinical training prior to incorporating this technique into clinical practice. The authors also pointed out a few limitations including the retrospective nature and the small sample size of the study. With all of that being said, Dr. Al-Kaisy and his research team have provided an innovative and valuable DRG approach to the pain medicine community and should be commended for their initial success. 


\section{REFERENCES}

1. Al-Kaisy A, Royds J, Costanzi M, et al. Effectiveness of "transgrade" epidural technique for dorsal root ganglion stimulation. A retrospective singe center case series for chronic focal neuropathic pain. Pain Physician. Manuscript PAINPHYSICIAN-D-19-00078R2.

2. Deer TR, Pop JE, Lamer TJ, et al. The Neuromodulation Appropriateness
Consensus Committee on Best Practices for Dorsal Root Ganglion Stimulation. Neuromodulation 2018.

3. Abd-Elsayed A, Lee S, King C. Retrograde placement of spinal cord stimulator leads for treating resistant pelvic pain. Saudi J Anaesth 2017:11: 336-367.

4. De Andres J, Perotti L, Villanueva-Perez
VL, Ansensio-Samper JM, Fabregat-Cid G. Role of lumbosacral retrograde neuromodulation in the treatment of painful disorders. Pain Physician 2013:16: 145-153.

5. Perper Y. Retrograde spinal cord stimulator lead placement for right $\mathrm{L}_{5}$ radiculopathy. Pain Med 2012:13: 733-734. 